\title{
Consumo larval y preferencia de oviposición de Gonipterus pulverulentus (Coleoptera: Curculionidae) asociados a distintas especies de Eucalyptus (Myrtaceae)
}

\author{
Larval consumption and oviposition preference of Gonipterus pulverulentus \\ (Coleoptera: Curculionidae) among different species of Eucalyptus (Myrtaceae)
}

\author{
María Riquelme Virgala a,c*, Gisela Di Silvestro a , Carmen Martínez ${ }^{+}$, Marina Santadino a,c, \\ Teresita Poretti b,c, Agustina Ansa a,c, Carlos Coviella b,c
}

\footnotetext{
*Autor de correspondencia: ${ }^{a}$ Universidad Nacional de Luján, Departamento de Tecnología, Laboratorio de Zoología Agrícola, Luján, Buenos Aires, Argentina, tel.: +54-02323-423979 int 1943, mriquelme@agro.uba.ar

${ }^{\mathrm{b}}$ Universidad Nacional de Luján, Departamento de Ciencias Básicas, Buenos Aires, Argentina.

c Universidad Nacional de Luján, Programa de Ecología Terrestre, INEDES-CONICET, Buenos Aires, Argentina.
}

\begin{abstract}
SUMMARY
Eucalyptus trees are among the most important forest resources in Argentina. The weevils of the genus Gonipterus are specialist insects that feed on their leaves. The objectives of this work were to estimate larval consumption and to evaluate the oviposition preference of $G$. pulverulentus among different species of Eucalyptus. Developmental time, consumption, final weight and some nutritional indexes were evaluated from cohorts of larvae grown on five different Eucalyptus species. Ovipositional preference was evaluated by a multiple choice assay. Five couples were placed in the center of a cage, together with a branch of each Eucalyptus species. The oothecae and the eggs inside them were counted for 12 days. Larval developmental times did not differ among the Eucalyptus species tested, although differences in final weight were observed. The larvae that consumed E. maidenii almost doubled the weight of those fed on E. camaldulensis. Maximum consumption was observed on larvae feeding on E. globulus and it was significantly higher than that of larvae feeding on the $E$. viminalis. The efficiency of conversion of ingested food of the larvae fed with $E$. viminalis was significantly larger than that recorded on E. globulus and E. camaldulensis. The choice experiment shows that females had no ovipositional preference. These results indicate that E. viminalis and E. camaldulensis are the species of higher and lower nutritional quality respectively for the development of G. pulverulentus.
\end{abstract}

Key words: eucalyptus snout beetle, nutritional indices, forest health.

\section{RESUMEN}

La producción de Eucalyptus spp. constituye uno de los recursos forestales más importantes en Argentina. Los gorgojos del género Gonipterus se alimentan específicamente de las hojas de estos árboles. El objetivo de este trabajo fue estimar el consumo de las larvas y evaluar la preferencia de oviposición de las hembras de G. pulverulentus, entre diferentes especies de Eucalyptus. Se determinaron: tiempo de desarrollo, consumo, peso final y algunos índices nutricionales de una cohorte de larvas criadas en cinco especies de eucalipto. La preferencia de oviposición se evaluó mediante un experimento de opción múltiple. Cinco parejas fueron colocadas en el centro de una jaula junto con una rama de cada eucalipto y durante 12 días se contabilizaron las ootecas y los huevos dentro de ellas. El tiempo de desarrollo larval no difirió entre las especies de eucalipto evaluadas, aunque sí se observaron diferencias en el peso final de las larvas; las que consumieron E. maidenii casi duplicaron el peso de las que se alimentaron sobre E. camaldulensis. Los máximos consumos estuvieron asociados a E. globulus y E. camaldulensis, los que fueron significativamente superiores al de E. viminalis. La eficiencia de conversión del alimento ingerido de las larvas alimentadas con $E$. viminalis fue significativamente mayor que la registrada para E. globulus y E. camaldulensis. Las hembras no presentaron preferencia de oviposición por ninguna de las especies evaluadas. Estos resultados indican que E. viminalis y E. camaldulensis son las especies de mayor y menor calidad nutricional, respectivamente, para el desarrollo de G. pulverulentus.

Palabras clave: gorgojo del eucalipto, índices nutricionales, sanidad forestal.

\section{INTRODUCCIÓN}

En Argentina, la forestación comercial con especies del género Eucalyptus L'Hèr ocupa el segundo lugar en importancia luego de las coníferas, siendo sus principales destinos la industria de papel y de madera sólida. La superficie ocupada por montes de Eucalyptus spp. alcanza las 255.000 ha, lo cual representa un $22,8 \%$ de la superfi- 
cie total implantada para producción forestal, cultivándose principalmente las siguientes especies y sus híbridos: E. camaldulensis Dehnh, E. globulus Labill, E. grandis Hill ex Maiden, E. tereticornis Smith, E. saligna Smith y E. viminalis Labill (UCAR 2015). La sanidad de estos árboles, originarios del continente oceánico, se caracteriza por estar asociada a plagas del mismo origen, cuyo número ha ido creciendo en los últimos años debido a la expansión mundial de las plantaciones y a la comercialización global de productos forestales (Paine et al. 2011).

Entre las primeras especies fitófagas que expandieron su distribución por fuera de su lugar de origen se encuentran los gorgojos del género Gonipterus Schoengerr. Las especies presentes en Argentina son Gonipterus platensis Marelli y G. pulverulentus Lea, las cuales fueron registradas por primera vez en el año 1925 (Marelli 1927) y rápidamente se dispersaron por el territorio asociadas a la presencia de eucaliptos. El daño que producen se debe a la alimentación de las larvas y de los adultos que consumen específicamente las hojas de los árboles de este género, pudiendo desfoliar completamente los brotes nuevos en caso de ataques severos (EPPO 2005). En su lugar de origen y en muchos donde estos gorgojos han sido introducidos, incluida la Argentina, se encuentra su parasitoide oófago específico, Anaphes nitens Girault (Hymenoptera, Mymaridae), controlando sus poblaciones. Sin embargo, bajo determinadas condiciones se ha visto un crecimiento poblacional asociado a mayores daños en diferentes especies de eucaliptos (Mapondera et al. 2012, Medeiros de Souza et al. 2016).

Existen numerosos estudios sobre la biología, el comportamiento y el control biológico de G. platensis (Cordero-Rivera et al. 1999, Cordero-Rivera y SatolamazzaCarbone 2000, De Oliveira 2006, Santolamazza-Carbone et al. 2006). Comparativamente, son escasos los antecedentes sobre G. pulverulentus (Freitas 1991ab), probablemente debido a que la distribución de esta especie por fuera de su lugar de origen se limita a Brasil, Uruguay y Argentina, no habiéndose registrado aún en el continente europeo, donde se la considera una plaga cuarentenaria en varios países (EPPO 2005). Además, durante varios años ambos gorgojos fueron considerados una misma especie, hasta que Rosado-Neto y Marques (1996) las diferenciaron a través de las características de las larvas y de la genitalia del macho. Por otro lado, recientes estudios taxonómicos basados en análisis moleculares y en la morfología del aedeagus, indicaron que Gonipterus scutellatus es un complejo de al menos 10 especies crípticas, de las cuales G. pulverulentus y G. platensis, anteriormente citadas como G. gibberus y G. scutellatus, respectivamente, son las especies presentes en Sudamérica (Mapondera et al. 2012, Medeiros de Souza 2016).

La ecología nutricional es el estudio del efecto de la calidad del alimento sobre la fisiología y el comportamiento de los individuos. La estimación del crecimiento, el consumo y el tiempo de desarrollo, son variables frecuentemente utilizadas para evaluar la calidad nutricional del alimento en los insectos herbívoros (Awmack y Leather 2002). Por otro lado, la teoría de la oviposición óptima predice que las hembras seleccionarán para oviponer aquellas plantas que maximicen el desarrollo de su descendencia (Thompson 1998, Awmack y Leather 2002). Ambos aspectos de la biecología de una plaga, pueden utilizarse para describir su comportamiento frente a diferentes especies de plantas de importancia comercial.

Una hipótesis que se plantea en este trabajo es que las especies de Eucalyptus poseen diferente calidad nutricional para G. pulverulentus, lo que afecta a la eficiencia de consumo y crecimiento de las larvas. Además, se espera que las hembras de este gorgojo seleccionen a determinadas especies de Eucalyptus para oviponer y que esto esté en relación con la calidad nutricional de las mismas. El presente trabajo tiene como objetivo evaluar la calidad nutricional durante el desarrollo larval y la preferencia de oviposición de las hembras de Gonipterus pulverulentus, asociados a varias especies de Eucalyptus de importancia económica en Argentina.

\section{MÉTODOS}

Los estudios se desarrollaron en el laboratorio de Zoología Agrícola de la Universidad Nacional de Luján. Los insectos utilizados se obtuvieron de la cría llevada a cabo en el mismo laboratorio, cuyos individuos inicialmente fueron recolectados de montes ubicados en las cercanías de la localidad de Balcarce, Provincia de Buenos Aires ( $37^{\circ} 83^{\prime}$ latitud Sur y $8^{\circ} 25^{\prime}$ longitud Oeste), los que fueron alimentados con hojas y brotes frescos de E. dunnii Maiden, especie que no se utilizó en los estudios.

Consumo larval. El ensayo se desarrolló en una cámara de cría JEIO Tech ${ }^{\circledR}$ GC-300/1000 bajo las siguientes condiciones: $24 \pm 1{ }^{\circ} \mathrm{C}, 60 \pm 10 \% \mathrm{HR}$, fotoperíodo $14 \mathrm{~L}: 10 \mathrm{O}$. Para obtener la cohorte de individuos necesaria para el ensayo, 50 ootecas fueron retiradas de la cría y ubicadas de a 10 en potes plásticos de $50 \mathrm{~cm}^{3}$ tapados con tela de voile. Una vez eclosionadas, se registró el peso inicial de 10 larvas por especie de Eucalyptus, las que fueron colocadas individualmente en una caja de Petri sobre una hoja previamente pesada (hoja con larva $=\mathrm{HL}$ ). Igual número de hojas se dejaron sin larvas (hojas testigo $=$ HT) para corregir el consumo por la pérdida de humedad, según la metodología propuesta por Cordero-Rivera y Santolamazza-Carbone (2000). Las hojas con larvas y sus correspondientes hojas testigo fueron seleccionadas de a pares con menos de un $10 \%$ de diferencia en su peso inicial. Se utilizaron hojas adultas recientemente cortadas de E. globulus, E. maidenii F. Muell., E. tereticornis, E. camandulensis y E. viminalis, recolectados del arboreto de la Universidad Nacional de Luján $\left(34^{\circ} 36^{\prime}\right.$ latitud Sur y 59 $04^{\circ}$ ' longitud Oeste). Cada dos días, las hojas fueron retiradas, pesadas y renovadas hasta el completo desarrollo de la larva. Las 
larvas y las hojas fueron pesadas utilizando una balanza analítica Ohaus Explorer ${ }^{\circledR}(\mathrm{d}=0,01 \mathrm{mg})$. El consumo se estimó por la fórmula [1]:

$$
C=(P i H L-P f H L) \times\left[1-\left(\frac{(P i H T-P f H T)}{P i H T}\right)\right]
$$

Donde, $C=$ Consumo (g). PiHL = Peso inicial de la hoja con larva (g). $F f H L=$ Peso final de la hoja con larva (g). $P i H T=$ Peso inicial de la hoja testigo (g). PfHT $=$ Peso final de la hoja testigo $(\mathrm{g})$.

El consumo total se obtuvo con la sumatoria de los consumos obtenidos cada dos días. Con los datos obtenidos se obtuvieron los siguientes índices nutricionales (Rossetti et al. 2008):

$$
\begin{gathered}
\text { TRCr }=\frac{P f-P i}{P g \times T} \\
T R C o=\frac{C}{P g \times T} \\
E C A i=\frac{(P f-P i)}{C} \times 100
\end{gathered}
$$

Donde, $T R C r=$ Tasa relativa de crecimiento $\left(\mathrm{mg} \mathrm{mg}^{-1}\right.$ día $\left.^{-1}\right) . T R C o=$ Tasa relativa de consumo $\left(\mathrm{mg} \mathrm{mg}^{-1}\right.$ día $\left.^{-1}\right)$. $E C A i=$ Eficiencia de consumo del alimento ingerido (\%). $P f=$ Peso final de la larva (mg). $P i=$ Peso inicial de la larva $(\mathrm{mg}) . P g=$ (Media geométrica del peso larval) $(\mathrm{mg})$ $=\sqrt{P i \times P f} . C=$ Consumo total $(\mathrm{mg}) . T=$ Período de tiempo (día).

Preferencia de oviposición. El ensayo se desarrolló en jaulas de cría (BugDorm-1 ${ }^{\circledR}$ ) ubicadas en un cuarto con condiciones semicontroladas $\left(24 \pm 3{ }^{\circ} \mathrm{C}, 60 \pm 15 \% \mathrm{HR}\right.$, fotoperíodo natural correspondiente al mes de octubre en las coordenadas $34^{\circ} 58^{\prime}$ latitud Sur y $59^{\circ} 09^{\prime}$ longitud Oeste).

Se realizó un experimento de opción múltiple con las mismas especies de Eucalyptus utilizadas en el ensayo anterior. En tres jaulas de cría se colocaron ramas de $20 \mathrm{~cm}$ recientemente cortadas, a las que se les dejaron ocho hojas expandidas, de las cinco especies de eucalipto, con su extremo sumergido en un recipiente con agua para evitar su deshidratación. La disposición dentro de la jaula fue al azar y equidistante entre sí. Al inicio del ensayo, cinco parejas de adultos de 15 a 30 días de edad obtenidos de la cría en laboratorio, fueron colocadas en el centro de la jaula. Cada dos días y durante 12 días, las ramas fueron renovadas y las ootecas obtenidas fueron disectadas para registrar el número total de huevos. Las mismas ramas sirvieron de alimentación para los adultos.

Análisis estadístico. En el primer estudio se siguió un diseño completamente aleatorizado con cinco tratamientos (especies de Eucalyptus) y 10 repeticiones (larvas). Las variables consumo larvario total, peso final de la larva y los índices nutricionales fueron analizadas con un ANDEVA de un factor y cuando resultó significativo, las medias fueron separadas con la prueba de Games-Howell. El tiempo de desarrollo larval fue analizado con la prueba de Kruskall-Wallis por no cumplirse el supuesto de normalidad (Kolmogorov-Smirnov, $P<0,05$ ).

En el ensayo de preferencia de oviposición, las variables número total de ootecas y número de huevos por ooteca fueron analizadas con un ANDEVA para un diseño en bloques completamente aleatorizados con tres repeticiones (jaulas). Se utilizó el programa estadístico IBM SPSS Statistics (versión 21).

\section{RESULTADOS}

El tiempo de desarrollo larval no difirió estadísticamente entre las especies de eucalipto (prueba KruskalWallis, $P=0,349$ ) (cuadro 1). La duración mínima y máxima registradas para este ensayo fue de 17 y 36 días, correspondientes a las larvas alimentadas con E. globulus y E. maidenii respectivamente. Sin embargo, se observaron diferencias significativas en el peso final de las larvas alimentadas con distintas especies de eucalipto $\left(\mathrm{F}_{4,33}=5,23\right.$; $P=0,002)$. Las larvas que consumieron $E$. maidenii casi duplicaron el peso de las que ingirieron $E$. camaldulensis al final del período (cuadro 1).

El consumo total durante el período larval varió significativamente entre especies de eucalipto $\left(\mathrm{F}_{4,33}=6,76\right.$; $P<0,001)$. El máximo consumo estuvo asociado a E. globulus $(1,15 \pm 0,09 \mathrm{~g})$, seguido por E. camaldulensis

Cuadro 1. Tiempo de desarrollo larval (días) y peso final de la larva de Gonipterus pulverulentus alimentadas con diferentes especies de Eucalyptus.

Larval developmental time (days) and larval final weight of Gonipterus pulverulentus larvae fed with different Eucalyptus species.

\begin{tabular}{lccc}
\hline \multicolumn{1}{c}{ Especie } & $\mathrm{N}^{\mathrm{a}}$ & $\begin{array}{c}\text { Período larval }(\mathrm{d}){ }^{\mathrm{b}} \text {. } \\
\text { Media } \\
\text { (error estándar) }\end{array}$ & $\begin{array}{c}\text { Peso final }(\mathrm{mg})^{\mathrm{c}} . \\
\text { Media } \\
\text { (error estándar) }\end{array}$ \\
\hline E. maidenii & 10 & $23,10(1,32) \mathrm{a}$ & $152,79(8,57) \mathrm{a}$ \\
E. globulus & 7 & $26,14(3,02) \mathrm{a}$ & $111,92(12,08) \mathrm{ab}$ \\
E. tereticornis & 7 & $25,71(1,85) \mathrm{a}$ & $123,56(9,15) \mathrm{ab}$ \\
E. camaldulensis & 6 & $25,40(2,11) \mathrm{a}$ & $86,28(16,54) \mathrm{b}$ \\
E. viminalis & 8 & $21,50(1,05) \mathrm{a}$ & $135,00(9,52) \mathrm{ab}$ \\
\hline
\end{tabular}

${ }^{a}$ Corresponde al número de larvas que completaron su desarrollo a partir de una cohorte inicial de diez (10).

${ }^{\mathrm{b}}$ Letras iguales indican que no hay diferencias significativas según la prueba de Kruskal Wallis $(P>0,05)$.

${ }^{c}$ Letras distintas indican diferencias significativas según la prueba de Games Howell $(P<0,05)$. 
$(0,89 \pm 0,035 \mathrm{~g})$, los que fueron significativamente superiores al consumo de $E$. viminalis $(0,66 \pm 0,056 \mathrm{~g})$ (figura 1$)$.

La tasa de crecimiento y la tasa de consumo (cuadro 2) no fueron afectadas por el alimento $\left(\mathrm{F}(\mathrm{TRCr})_{4,33}=2,27\right.$, $P=0,083$; Kruskal-Wallis, $P=0,310)$. Sin embargo, la eficiencia de conversión del alimento ingerido varió entre especies de Eucalyptus $\left(\mathrm{F}\left(\mathrm{ECA}_{\mathrm{i}}\right)_{4,33}=7,44, P<0,001\right)$. En el caso de las larvas que consumieron $E$. viminalis, la eficiencia de conversión fue significativamente mayor que la obtenida de las larvas que se alimentaron de E. globulus y E. camaldulensis (cuadro 2).

Preferencia de oviposición. Las hembras de G. pulverulentus colocaron ootecas en todas las especies de Eucalyptus, en un número que no fue significativamente diferente $\left(\mathrm{F}_{4,8}=0,54 ; P=0,714\right)$. Del mismo modo, el número medio de huevos por ooteca no varió significativamente $\left(\mathrm{F}_{4,8}=2,35 ; P=0,141\right)$ (cuadro 3$)$.

\section{DISCUSIÓN}

La cantidad y calidad de alimento pueden influir en la tasa de crecimiento y desarrollo, el peso final, la supervivencia, así como en la fecundidad y la capacidad de dispersión de los adultos. En este trabajo, si bien Gonipterus pulverulentus completó su desarrollo larval alimentándose de todas las especies de Eucalyptus evaluadas y en un tiempo que no difirió entre tratamientos, otros parámetros fueron afectados por el alimento. La duración de la etapa larval fue además similar a la estimada para esta especie alimentada con E. saligna Smith (Freitas 1991b) y para G. platensis (Santolamazza-Carbone et al. 2006).

El consumo y el peso final de las larvas variaron significativamente según la fuente de alimento. Los mayores consumos fueron registrados sobre las especies E. globulus y E. camaldulensis, valores significativamente superiores al observado para E. viminalis, mientras que el mayor peso final fue registrado en las larvas alimentadas con E. maidenii, las cuales casi duplicaron el peso medio alcanzado en el tratamiento con E. camaldulensis. Algunos estudios sugieren que el menor tamaño de los individuos podría afectar el desempeño reproductivo de los adultos y volverlos más vulnerables al ataque de enemigos naturales (Greenberg et al. 2005, Zabala 2010). Freitas (1991a) registró para esta especie un consumo de E. saligna y un peso final de las larvas menores a los estimados en todos los tratamientos en este trabajo $(0,44 \mathrm{~g} \mathrm{y} 73,7 \mathrm{mg}$, respectivamente), lo que podría indicar una menor calidad nutricional de esta especie de Eucalyptus.

Si bien la tasa de crecimiento y la tasa de consumo no variaron entre tratamientos, la eficiencia de conversión

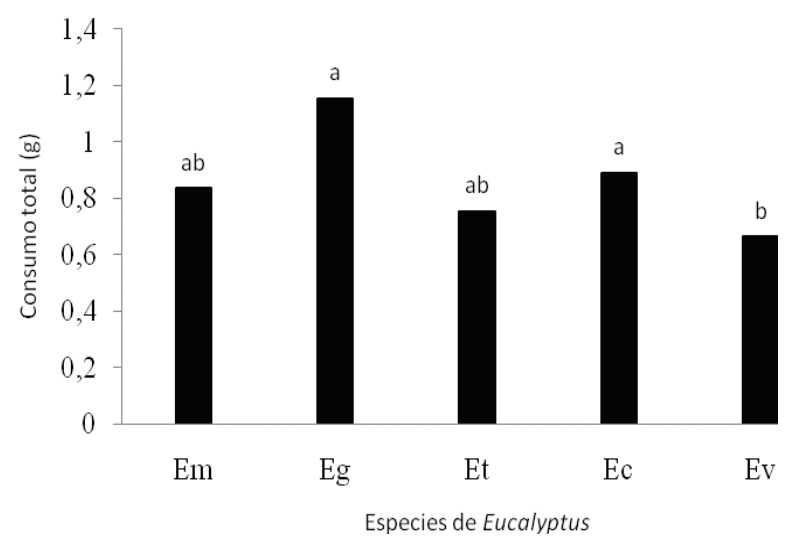

Figura 1. Consumo larval (g) de Gonipterus pulverulentus de diferentes especies de Eucalyptus. Em: Eucalyptus maidenii; Eg: E. globulus; Et: E. tereticornis; Ec: E. camaldulensis; Ev: E. viminalis. Letras distintas indican diferencias significativas según la prueba de Games Howell $(P<0,05)$.

Larval consumption (g) of different Eucalyptus species by Gonipterus pulverulentus.

Cuadro 2. Índices nutricionales de larvas de Gonipterus pulverulentus alimentadas con diferentes especies de Eucalyptus.

Nutritional indices of Gonipterus pulverulentus larvae fed with different Eucalyptus species.

\begin{tabular}{lccc}
\hline Especie & $\begin{array}{c}\text { TRCr }\left(\mathrm{mg} \mathrm{mg}^{-1} \text { día }^{-1}\right)^{\mathrm{a}} . \\
\text { Media (error estándar) }\end{array}$ & $\begin{array}{c}\text { TRCo }\left(\mathrm{mg} \mathrm{mg}^{-1} \text { día }^{-1}\right)^{\mathrm{b}} . \\
\text { Media (error estándar) }\end{array}$ & $\begin{array}{c}\text { ECAi }(\%)^{\mathrm{c}} . \\
\text { Media (error estándar) }\end{array}$ \\
\hline E. maidenii & $0,49(0,06) \mathrm{a}$ & $2,77(0,47) \mathrm{a}$ & $18,71(1,41) \mathrm{ab}$ \\
E. globulus & $0,30(0,04) \mathrm{a}$ & $3,16(0,38) \mathrm{a}$ & $10,06(1,53) \mathrm{b}$ \\
E. tereticornis & $0,40(0,08) \mathrm{a}$ & $2,67(0,85) \mathrm{a}$ & $17,83(2,85) \mathrm{ab}$ \\
E. camaldulensis & $0,27(0,07) \mathrm{a}$ & $2,84(0,44) \mathrm{a}$ & $9,70(2,35) \mathrm{b}$ \\
E. viminalis & $0,43(0,05) \mathrm{a}$ & $2,11(0,21) \mathrm{a}$ & $20,26(0,62) \mathrm{a}$ \\
\hline
\end{tabular}

TRCr: tasa de crecimiento; TRCo: tasa de consumo; ECAi: eficiencia de conversión del alimento ingerido.

${ }^{a}$ Letras iguales indican que no hay diferencias significativas en el ANDEVA $(P>0,05)$.

${ }^{\mathrm{b}}$ Letras iguales indican que no hay diferencias significativas según la prueba de Kruskal Wallis $(P>0,05)$.

${ }^{\mathrm{c}}$ Letras distintas indican diferencias significativas según la prueba de Games Howell $(P<0,05)$. 
Cuadro 3. Número de ootecas y de huevos por ooteca puestos por hembras de Gonipterus pulverulentus en diferentes especies de Eucalyptus.

Number of oothecae and number of eggs per ootheca laid by Gonipterus pulverulentus females on different Eucalyptus species.

\begin{tabular}{lcc}
\hline Especie & $\begin{array}{c}\text { Número de ootecas }{ }^{\mathrm{a}} \text {. } \\
\text { Media } \\
\text { (error estándar) }\end{array}$ & $\begin{array}{c}\text { Huevos/ooteca }{ }^{\mathrm{a}} . \\
\text { Media } \\
\text { (error estándar) }\end{array}$ \\
\hline E. maidenii & $16,67(4,26) \mathrm{a}$ & $2,61(0,43) \mathrm{a}$ \\
E. globulus & $10,00(4,41) \mathrm{a}$ & $2,89(0,30) \mathrm{a}$ \\
E. tereticornis & $17,67(10,30) \mathrm{a}$ & $2,86(0,10) \mathrm{a}$ \\
E. camaldulensis & $18,33(8,44) \mathrm{a}$ & $2,62(0,12) \mathrm{a}$ \\
E. viminalis & $12,33(2,48) \mathrm{a}$ & $2,03(0,21) \mathrm{a}$ \\
\hline
\end{tabular}

${ }^{a}$ Letras iguales dentro de una misma columna indican que no hay diferencias significativas en el ANDEVA $(P>0,05)$.

del alimento ingerido fue significativamente diferente. La máxima eficiencia presentada por las larvas alimentadas con E. viminalis concuerda con que esta especie fue la menos consumida, pero el peso final alcanzado por las larvas solo fue superado por las que consumieron $E$. maidenii. Por el contrario, las larvas alimentadas con E. camaldulensis mostraron la menor eficiencia, debido a que presentaron el menor peso promedio y un consumo solo superado, aunque no significativamente, por las que consumieron E. globulus. Las características físicas y la composición química de las hojas, son factores que influyen en la interacción plantaherbívoro en los insectos defoliadores (Zabala 2010). En particular, las hojas del género Eucalyptus contienen gran cantidad de compuestos secundarios como aceites esenciales, taninos y otros fenoles, y además poseen un follaje esclerófilo con elevado contenido de fibra, ceras cuticulares y baja concentración de nitrógeno (Ohmart y Edwards 1991). En consecuencia, las larvas podrían aumentar el consumo principalmente para obtener el nitrógeno suficiente para cumplir su desarrollo (Ohmart et al. 1985). Por otro lado, Zabala (2010) propone que una de las defensas de las plantas contra la herbivoría son las proteínas que inhiben la actividad de las enzimas (proteasas) encargadas de digerir las proteínas del alimento. Como consecuencia, la asimilación de aminoácidos por parte del insecto disminuye, generándose individuos de menor tamaño, a pesar de que en algunos casos las larvas tienden a compensar la disminución de la asimilación de aminoácidos aumentando el consumo de área foliar. Resultados similares obtuvieron Cordero-Rivera y Santolamazza-Carbone (2000) quienes observaron para G. platensis un valor de consumo de E. globulus de 1,24 g y uno marcadamente mayor de $E$. citriodora $(3,49 \mathrm{~g})$, especie que estos autores consideraron menos palatable, ya que registraron además una menor supervivencia en la cohorte de larvas alimentadas con esta especie de Eucalyptus.
Las hembras ovipusieron en todas las especies evaluadas, y tanto el número de ootecas como el promedio de huevos/ooteca no presentaron diferencias entre tratamientos. Freitas (1991b) encontró que esta especie coloca ootecas con uno a seis huevos, con una moda de tres huevos por ooteca, valor similar al promedio observado en este trabajo (2,60 huevos/ooteca). Algunos autores afirman que la edad de las hojas es más importante en la elección para oviponer y alimentarse que otros factores como el origen genético de las mismas (Steinbauer et al. 1998, Gherlenda et al. 2016). Esta preferencia por hojas de menor edad podría ser el resultado de su mayor concentración de nutrientes particularmente nitrogenados y sus menores defensas físicas, en particular en árboles con follaje esclerófilo como el de los Eucalyptus spp., lo que podría favorecer el desarrollo de las larvas (Gherelenda et al. 2016). Por su parte, Bouwer et al. (2014) mostraron que las sustancias volátiles emitidas por el hospedante juegan un rol significativo en la elección de la especie de Eucalyptus por parte de las hembras de Gonipterus sp.

La relación entre la preferencia de oviposición de las hembras y el desarrollo de su descendencia, ha sido motivo de diversos estudios e hipótesis (Thompson 1998, Awmack y Leather 2002). En aquellas especies que presentan una estrecha relación entre la preferencia de oviposición de las hembras y el desempeño de su descendencia, el principal factor que determina esta discriminación está basado frecuentemente en la calidad nutricional de la planta hospedante. En este trabajo, la diferente eficiencia de conversión del alimento ingerido según la especie de Eucalyptus, podría sugerir una diferente calidad nutricional en las especies evaluadas que, sin embargo, no se vio reflejada en la preferencia de oviposición de las hembras. Si bien se ha comprobado para otras especies que se alimentan de eucalipto una relación positiva entre ambos factores (Steinbauer 2002, Martínez et al. 2016), en otros casos no se evidencia tan claramente, sugiriendo la intervención de otros factores en la preferencia de oviposición, como el vigor de la planta, la competencia intra e interespecífica y el espacio libre de enemigos naturales (Nahrung y Allen 2003, Heisswolf et al. 2005). Particularmente para el género Gonipterus, Newete et al. (2011) encontraron que la alimentación y oviposición en el campo parecen ser más selectivas que lo que se observa en estudios de laboratorio y concluyeron que la supervivencia larval y la preferencia de oviposición para estos gorgojos no necesariamente se correlacionan.

\section{CONCLUSIONES}

Los resultados de este estudio aportan nuevos conocimientos sobre una especie del género Gonipterus escasamente estudiada en el mundo. Si bien estos gorgojos pudieron completar su desarrollo larval en todas las especies de Eucalyptus evaluadas, los resultados confirman la hipótesis de que la diferente calidad nutricional repercute en la eficiencia de crecimiento y desarrollo de las larvas. 
Por el contrario, las hembras no son selectivas en su preferencia de oviposición, indicando que podrían influir otros factores en la elección del material además de la calidad nutricional como alimento para su descendencia.

\section{REFERENCIAS}

Awmack CS, SR Leather. 2002. Host plant quality and fecundity in herbivorous insects. Annual Review of Entomology 47: 817-844.

Bouwer MC, B Slipers, MJ Wingfield, ER Rohwer. 2014. Chemical signatures affecting host choice in the Eucalyptus herbivore, Gonipterus sp. (Curculionidae: Coleoptera). Arthroppod-Plant Interaction 8:439-451.

Cordero-Rivera A, S Santolamazza-Carbone, JA Andrés. 1999. Life cycle and biological control of the Eucalyptus snout beetle (Coleoptera, Curculionidae) by Anaphes nitens (Hymenoptera, Mymaridae) in north-west Spain. Agricultural and Forest Entomology 1: 103-109.

Cordero-Rivera A, S Santolamazza-Carbone. 2000. The effect of three species of Eucalyptus on growth and fecundity of the Eucalyptus Snout Beetle (Gonipterus scutellatus, Coleoptera, Curculionidae). Forestry 73(1): 21-29.

De Oliveira NC. 2006. Biología de Gonipterus scutellatus (Coleoptera: Curculionidae) em Eucalyptus spp. em diferentes temperaturas. Tesis Doutor em Agronomia (Proteção de Plantas). Campus de Botucatu, Brasil. Faculdade de Ciências Agronômicas, Universidade Estadual Paulista. 92 p.

Freitas S. 1991a. Observaçoes sobre a alimentaçao de Gonipterus gibberus (Boisduval, 1835) (Coleoptera, Curculionidae) em eucaliptos. Anais da Sociedade Entomologica do Brasil 20: 333-338.

Freitas S. 1991b. Biología de Gonipterus gibberus (Boisduval, 1835) (Coleoptera, Curculionidae) una praga do eucaliptos. Anais da Sociedade Entomologica do Brasil 20: 339-344.

Gherlenda AN, BD Moore, AM Haigh, SN Johnson, M Riegler. 2016. Insect herbivory in a mature Eucalyptus woodland canopy depends on leaf phenology but not $\mathrm{CO}_{2}$ enrichment. BMC Ecology 16:47. DOI 10.1186/s12898-016-0102-z

Greenberg SM, DW Spurgeon, T Sappington, M Setamou. 2005. Size-Dependent Feeding and Reproduction by Boll Weevil (Coleoptera: Curculionidae). Journal of Economic Entomology 98(3): 749-756.

Heisswolf A, E Obermaier, HJ Poethke. 2005. Selection of large host plants for oviposition by a monophagous leaf beetle: nutritional quality or enemy-free space? Ecological Entomology 30: 299-306.

Mapondera TS, T Burgess, M Matsuki, RG Oberprieler. 2012. Identification and molecular phylogenetics of the cryptic species of the Gonipterus scutellatus complex (Coleoptera: Curculionidae: Gonipterini). Australian Journal of Entomology 51: 175-188.

Marelli CA. 1927. El gorgojo de los eucaliptos hallado en la Argentina no es la especie originaria de Tasmania „Gonipterus scutellatus" Gyll. Revista del Museo de La Plata 30: 257-269.

Martínez G, MV Finozzi, G Cantero, R Soler, M Dicke, A González. 2016. Oviposition preference but not adult feeding preference matches with offspring performance in the bronze bug Thaumastocoris peregrinus. Entomologia Experimentalis et Applicata 163: 101-111.
Medeiros de Souza N, LR Junqueira, CF Wilcken, EP Soliman, M Bueno de Camargo, MA Nickele, L Rodriguez Balbosa. 2016. Ressurgencia de uma antiga ameaca: Gorgulhodo-eucalipto Gonipterus platensis (Coleoptera: Curculionidae). Circular Técnica IPEF 209 20p. Consultado 10 mar. 2017. disponible en https://www.researchgate. net/profile/Carlos_Wilcken/publication/309126060_Ressurgencia_de_uma_antiga_ameaca_Gorgulho_do_Eucalipto_Gonipterus_platensis_Coleoptera_Curculionidae/ links/57fff30c08aec3e477eac84b/Ressurgencia-de-umaantiga-ameaca-Gorgulho-do-Eucalipto-Gonipterus-platensis-Coleoptera-Curculionidae.pdf

Nahrung HF, GR Allen. 2003. Intra-plant host selection, oviposition preference and larval survival of Chrysophtharta agricola (Chapuis) (Coleoptera: Chrysomelidae:Paropsini) between foliage types of a heterophyllous host. Agricultural and Forest Entomology 5: 155 \pm 162 .

Newete SW, RG Oberprieler, MJ Byrne. 2011. The host range on Eucalyptus weevil, Gonipterus "scutellatus" Gyllenhal (Coleoptera: Curculionidae) in South Africa. Annals of Forest Science 68: 1005-1013. DOI: 10.1007/s13595-0110108-9.

EPPO (European and Mediterranean Plant Protection Organization, FR). 2005. Gonipterus gibberus and Gonipterus scutellatus. Bulletin EPPO 35: 368-370. Consultado 25 oct. 2014. Disponible en https://www.eppo.int/QUARANTINE/data_sheets/insects/DS_Gonipterus_spp.pdf

Ohmart CP, PB Edwards. 1991. Insect herbivory on Eucalyptus. Annual Review of Entomology 36: 637-657.

Ohmart CP, LG Stewart, JR Thomas. 1985. Effects of food quality, particularly nitrogen concentrations, of Eucalyptus blakelyi foliage on the growth of Paropsisatomaria larvae (Coleoptera: Chrysomelidae). Oecologia 65: 543-549.

Paine TD, MJ Steinbauer, SA Lawson. 2011. Native and Exotic Pests of Eucalyptus: A Worldwide Perspective. Annual Review of Entomology 56: 181-201.

Rosado-Neto GH, MI Marques. 1996. Características do adulto, genitália e formas imaturas de Gonipterus gibberus Boisduval e G. scutellatus Gyllenhal (Coleoptera, Curculionidae). Revista Brasileira de Zoologia13: 77-90.

Rossetti MR, MT Defagó, MC Carpinella, SM Palacios, G Valladares. 2008. Actividad biológica de extractos de Melia azedarach sobre larvas de Spodoptera eridiana (Lepidoptera: Noctuidae). Revista de la Sociedad Entomológica Argentina 67(1-2): 115.125.

Santolamazza-Carbone S, A Rodríguez-Illamola, A CorderoRivera. 2006. Thermal requirements and phenology of the Eucalyptus snout beetle Gonipterus scutellatus Gyllenhal. Journal of Applied Entomology 130(6-7): 368-376.

Steinbauer M, A Clarke, J Madden.1998. Oviposition preference of a Eucalyptus herbivore and the importance of leaf age on interspecific host choice. Ecological Entomology 23: 201-206.

Steinbauer M. 2002. Oviposition preference and neonate performance of Mnesampela privata in relation to heterophylly in Eucalyptus dunnii and E. globulus. Agricultural and Forest Entomology 4: 245-253.

Thompson J. 1998. Evolutionary ecology of the relationship between oviposition preference and performance of offspring in phytophagous insects. Entomologia Experimentalis et Applicata 47: 3-14. 
UCAR (Unidad para el Cambio Rural, AR). 2015. Argentina: plantaciones forestales y gestión sostenible. Ministro de Agricultura, Ganadería y Pesca de la Nación Argentina 30p. Consultado 5 mar.2016. Disponible en https://drive.goo-
gle.com/file/d/0Bzc7dDyL0NwLT0VlcHdRdjNCYTA/ view

Zabala J. 2010. Respuestas inmunológicas de las plantas frente al ataque de insectos. Ciencia Hoy 20(117): 53-59.

Recibido: 06/04/18

Aceptado: 04/06/18 
